Schmerz 2020 $34: 281$

https://doi.org/10.1007/s00482-020-00452-2

Online publiziert: 23. April 2020

(c) Springer Medizin Verlag $\mathrm{GmbH}$, ein Teil von Springer Nature 2020

\author{
Jörn Lötsch ${ }^{1,2} \cdot$ Gerd Geisslinger ${ }^{1,2} \cdot$ Carmen Walter $^{2}$ \\ 'Institut für Klinische Pharmakologie, Goethe-Universität, Frankfurt am Main, Deutschland \\ ${ }^{2}$ Institutsteil Translationale Medizin und Pharmakologie (TMP), Fraunhofer-Institut für Molekularbiologie \\ und Angewandte Oekologie (IME), Frankfurt am Main, Deutschland
}

\title{
Schmerzmodelle und klinische Schmerzforschung sind kein Widerspruch
}

\section{Erwiderung}

Zum Leserbrief von Wörz R (2020) Wertvoller, doch begrenzter Aussagewert experimenteller Modelle. https://doi.org/10.1007/s00482020-00450-4

Originalbeitrag: Lötsch J, Geisslinger G, Walter $C$ (2019) Wissensgenerierung aus komplexen Datensätzen in der humanexperimentellen Schmerzforschung. Schmerz 33:S502-513. https://doi.org/10.1007/s00482-019-00412-5

Wir freuen uns, dass unser Beitrag in Der Schmerz zu weiteren Überlegungen anregt. Modelle sind in der Wissenschaft weit verbreitet, und ihre Definition impliziert bereits, dass sie eine vereinfachte Darstellung der Realität sind. In der Schmerzforschung sind präklinische Modelle Standard, und die experimentelle Forschung am Menschen hat wesentlich zu unserem Verständnis von Schmerz beigetragen. Darüber hinaus können neue Schmerztherapeutika, die sich in der Entwicklung befinden, unter Laborbedingungen vorgetestet werden, bevor sie zu früh eingesetzt werden und Schmerzpatienten im schlimmsten Fall eine adäquate Analgesie entziehen. Es gibt genügend Belege für korrekte Vorhersagen der klinischen Wirksamkeit. Weder modellbasierte noch klinische Forschung ist jedoch unfehlbar. Natürlich impliziert die Verwendung des Begriffs „Modell“ ein Bewusstsein für die vereinfachte, verkürzte Darstellung einer komplexeren Realität. Ausgelassene Teile davon können mithilfe anderer Modelle oder, wenn möglich, durch direktes Studium der Realität, im speziellen Fall von Schmerzpatienten, erforscht wer- den. Schmerzmodelle und klinische Schmerzforschung sind kein Widerspruch. Unser Beitrag konzentrierte sich auf Ersteres, und der Leserbrief ergänzt bekannte Grenzen der modellbasierten Schmerzforschung, wofür wir dankbar sind.

\section{Korrespondenzadresse}

\section{Prof. Dr. Dr. Jörn Lötsch}

Institut für Klinische Pharmakologie, GoetheUniversität

Theodor-Stern-Kai 7, 60590 Frankfurt am Main, Deutschland

j.loetsch@em.uni-frankfurt.de

Interessenkonflikt. J. Lötsch, G. Geisslinger und C. Walter geben an, dass kein Interessenkonflikt besteht. 